\title{
Structure determination of the motor domain of centromere associated protein $\mathrm{E}$
}

\author{
A. Shibuya ${ }^{1}$, N. Ogo ${ }^{2}$, J. Sawada ${ }^{2}$, A. Asai ${ }^{2}$, H. Yokoyama ${ }^{1}$, \\ ${ }^{1}$ Faculty of Pharmaceutical Sciences, Tokyo University of Science, 2641 Yamazaki, Noda, Chiba 278-8510, Japan., \\ ${ }^{2}$ Center for Drug Discovery, Graduate School of Pharmaceutical Sciences, University of Shizuoka, 52-1 Yada, Suruga-ku, Shizuoka \\ 422-8526, Japan. \\ yokoyama@rs.tus.ac.jp
}

Antimitotic agents that target mitotic kinesins such as centromere associated protein E (CENP-E), are expected to be more likely to work on dividing cells but not non-dividing cells. Thus, antimitotic agents that inhibit the functions of the kinesin motor domains will minimize toxicities to non-dividing cells, causing lower side effects [1].

The motor domain, located at the N-terminus of CENP-E, is the active site of ATPase activity. Up to now, the only one crystal structure of CENP-E motor domain in complex with MgADP has been reported [2]. It is difficult to perform rational drug designing by fragment-based drug discovery (FBDD) or structure-based drug design (SBDD) due to the lack of structural information about CENP-E. Therefore, it is necessary to determine the crystal structure of CENP-E motor domain in complex with its inhibitors.

Here, in order to elucidate the mechanism how CENP-E motor domain binds to its inhibitor, we tried to cocrystallize CENP-E motor domain in complex with its ligand, 3-chloro-4-isopropoxyl benzoic acid (CIBA), one of the ATP-competitive inhibitors, or GSK923295, one of the ATP-uncompetitive inhibitors. First, we crystallized CENP-E motor domain in complex with CIBA, and determined the structure at $1.9 \AA$ resolution (Figure 1). Endogenous ADP instead of CIBA was observed at the nucleotide-binding site, although ATP or ADP was not added. The determined structure of the CENP-E motor domain was compared with other kinesin motors. Based on the characteristic structure of CENP-E, the mechanism by which ADP is retained in CENP-E is discussed [3].

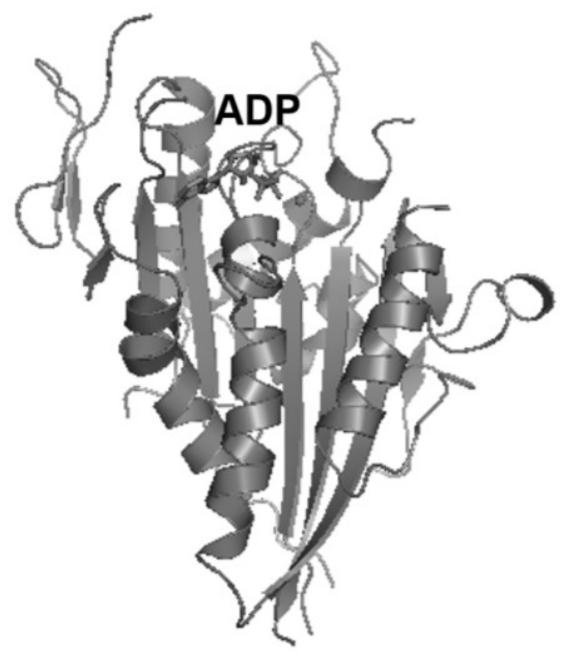

Figure 1. Front view of the CENP-E-MgADP of this study are shown in cartoon representation.

Next, in order to elucidate the structure in complex with an ATP analog, we tried to determine the structure of CENP-E motor domain in the presence of AMPPNP and $\mathrm{Mg}^{2+}$ at $1.8 \AA$ resolution. Crystals belong to space group $P 2_{1} 2_{1} 2$ with two molecules in the asymmetric unit. Structure refinement is now in progress.

[1] Sakowicz, R., Finer, J. T., Beraud, C., Crompton, A., Lewis, E., Fritsch, A., Lee, Y., Mak, J., Moody, R., Turincio, R., Chabala, J. C., Gonzales, P., Roth, S., Weitman, S. \& Wood, K. W. (2004). Cancer Res. 64, 3276-3280.

[2] Garcia-Saez, I., Yen, T., Wade, R. H. \& Kozielski, F. (2004). J. Mol. Biol. 340, 1107-1116.

[3] Shibuya, A., Ogo, N., Sawada, J., Asai, A., \& Yokoyama, H. (2021). Acta Crystallographica Section D, D77, $280-287$.

\section{Keywords: ATPase cycle; Kinesin; CENP-E}

We would highly appreciate the cooperation and support rendered to us by all the staff members of High Energy Accelerator Research Organization in Tsukuba. We thank all involved in holding this congress. 